\title{
Hematocrit-insensitive Absolute Blood Flow Rate Measurement in 0.5-mm-diameter Flow Channel Using MEMS-based Laser Doppler Velocimeter with Signal Modification for Detecting Beat Frequency from Broad Power Spectrum
}

\author{
Nobutomo Morita, ${ }^{1 *}$ Wataru Iwasaki, ${ }^{1}$ Hirofumi Nogami, ${ }^{2}$ Fumiya Nakashima, ${ }^{3}$ \\ Eiji Higurashi, ${ }^{4,5}$ and Renshi Sawada ${ }^{2}$ \\ ${ }^{1}$ Advanced Manufacturing Research Institute, National Institute of Advanced Industrial \\ Science and Technology (AIST), 807-1 Shuku-machi, Tosu, Saga 841-0052, Japan \\ ${ }^{2}$ Department of Mechanical Engineering, Faculty of Engineering, Kyushu University, \\ 744 Motooka, Nishi-ku, Fukuoka 819-0395, Japan \\ ${ }^{3}$ Graduate School of Systems Life Sciences, Kyushu University, 744 Motooka, Nishi-ku, Fukuoka 819-0395, Japan \\ ${ }^{4}$ Department of Precision Engineering, School of Engineering, The University of Tokyo, \\ 7-3-1 Hongo, Bunkyo-ku, Tokyo 113-8656, Japan \\ ${ }^{5}$ Research Center for Ubiquitous MEMS and Micro Engineering (UMEMSME), National Institute of Advanced \\ Industrial Science and Technology (AIST), 1-2-1 Namiki, Tsukuba, Ibaraki 305-8564, Japan
}

(Received May 28, 2018; accepted October 3, 2018)

Keywords: MEMS, LDV, frequency weighting, peak detection, blood flow

We applied our millimeter-scale laser Doppler velocimeter ( $\mu$-LDV) as an absolute blood flow rate sensor in a thin tube $(0.5 \mathrm{~mm}$ inner diameter) for precise control of the blood flow rate. We proposed a sequence of signal processing, frequency weighting modification, and peak detection, and succeeded in the detection of the beat signal induced by flow at the center of the tube. Flow rates of 20,40, and 60\% hematocrit [volume percentage of red blood cells (RBCs) in blood] were measured. The errors with respect to the hematocrit change were $14.2 \%$ for $58.9 \mu \mathrm{l} / \mathrm{min}, 5.1 \%$ for $235.6 \mu \mathrm{l} / \mathrm{min}$, and $3.6 \%$ for $471.1 \mu \mathrm{l} / \mathrm{min}$ (corresponding maximum flow velocity of $80 \mathrm{~mm} / \mathrm{s}$ ), whereas the error ranges determined by laser Doppler flowmetry (LDF) calculation, which is generally used for blood flow measurement by the laser Doppler effect, were 89.2, 42.7, and 19.5\%, respectively. The results show the potential application of $\mu-\mathrm{LDV}$ as an integrable absolute blood flow rate sensor on a microchannel such as a thin tube and micro-total analysis system ( $\mu$-TAS) for a wide hematocrit range.

\section{Introduction}

Microfluidic device technology is becoming increasingly important in the biomedical field. ${ }^{(1)}$ To date, a variety of microfluidic devices have been researched and developed toward realizing the lab-on-a-chip (LoC) and micro-total analysis system $(\mu-\mathrm{TAS})^{(2-6)}$ for various analyses and applications, such as liquid chromatography, ${ }^{(7)}$ protein separation, ${ }^{(8)}$

*Corresponding author: e-mail: morita.nobutomo@aist.go.jp

https://doi.org/10.18494/SAM.2018.2010 
rare metal extraction, ${ }^{(9)}$ Raman spectroscopy, ${ }^{(10)}$ DNA analysis, ${ }^{(11-14)}$ drug discovery, ${ }^{(15)}$ electrotaxis study, ${ }^{(16)}$ cancer biomarker detection, ${ }^{(17)}$ and hormone detection. ${ }^{(18)}$ Many of these applications require precise control of the flow rate. ${ }^{(19,20)}$

Several types of flow sensors for microfluidic devices have been developed, the measurement principle of which is based on thermal transport, ${ }^{(21)}$ electrical admittance change, ${ }^{(22)}$ fluid dynamical oscillation, ${ }^{(23)}$ optical imaging, ${ }^{(24)}$ and the laser Doppler effect. ${ }^{(25)}$ A laser Doppler velocimeter (LDV) achieves contactless measurement in real time without the need to add anything to the measurement target, because an LDV detects velocity from the Doppler shift of scattered light, which is proportional to the velocity of the measurement target. Thus, LDV has been used for blood flow measurement in both clinical and microfluidics research, for example, blood flow in a retinal vessel ${ }^{(26)}$ and experimental research on biorheology. ${ }^{(27,28)}$ However, only the total flow rate of blood is controlled by a syringe pump ${ }^{(11,29,30)}$ and the flow rate of each branched flow channel is not monitored. The application of LDV must be considered to guarantee blood flow rate measurement for further accurate analysis of blood samples. Unfortunately, an LDV requires a large optical system such as a microscope, giving rise to challenges such as difficulty of integration in a microfluidic device and the need for measurements of multiple adjacent points. Moreover, during the flow measurement of a thicker blood flow layer (i.e., $>400 \mu \mathrm{m}$ for $40 \%$ hematocrit), the power spectrum of the LDV signal is greatly broadened and the signal weakened owing to marked light scattering and absorption by red blood cells (RBCs), resulting in a difficulty in detecting beat frequency because of the Doppler shift required for the calculation of flow velocity. ${ }^{(31)}$ It is known that the blood flow rate can be evaluated by laser Doppler flowmetry (LDF) even with a broad power spectrum. However, in the LDF method, the measured value varies depending on the hematocrit value, ${ }^{(28)}$ which varies greatly among individuals (i.e., 40 to $54 \%$ for men, 36 to $48 \%$ for women ${ }^{(32)}$ ), thus preventing an absolute flow measurement of blood. We have developed a small MEMS-based LDV named micro-LDV ( $\mu$-LDV), which has the advantage of small size, high-speed response, and a wide range of measurable target materials, making its integration into a humanoid robotic fingertip as a slip sensor possible. ${ }^{(33)}$

In this paper, we report the absolute blood flow rate measurement using the newly developed $\mu$-LDV as an optical flow sensor system for accurate, multipoint, and real-time blood flow rate monitoring (Fig. 1). We propose a signal processing algorithm based on the frequency weighting method to detect beat frequency from a power spectrum signal with low intensity and a broad band as a result of light scattering by blood and high absorptivity. Then, we discussed the accuracy of the absolute flow rate measurement by the proposed method including the influence of hematocrit changes and compared it with the result calculated by the LDF method. 


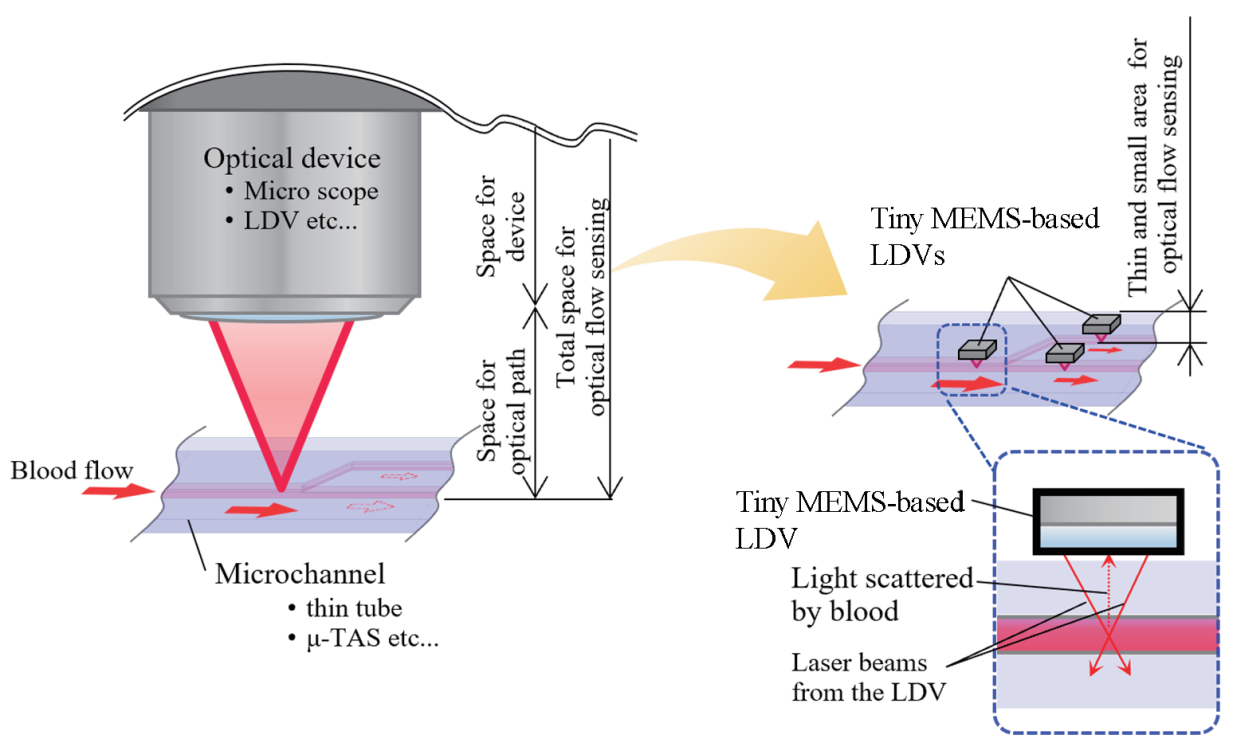

Fig. 1. (Color online) Schematic diagram of optical flow sensor system for accurate, multipoint, and real-time blood flow rate monitoring.

\section{Materials and Methods}

\subsection{Experimental setup}

Figure 2 shows the $\mu$-LDV and cover plate. The dimensions of the $\mu$-LDV with the cover plate are $2.8 \times 2.8 \mathrm{~mm}^{2}$ and $1.05 \mathrm{~mm}$ in thickness. The $\mu$-LDV mainly consists of the Si layer as the base material, the glass layer as the cover material, a laser diode (LD), and a photodiode (PD). The glass layer has two lenses for laser beam collimation. The $\mu$-LDV can irradiate two collimated laser beams that cross to form the measurement volume for laser Doppler velocimetry. Representative specifications of the $\mu$-LDV are listed in Table 1. Further details of the $\mu$-LDV, including the design, fabrication, and basic characteristics of velocity measurement, are described in our previous paper. ${ }^{(34)}$ In this work, we aimed to obtain a signal with a higher signal-to-noise ratio by attaching a cover plate with a pinhole onto the top surface of the $\mu$-LDV. The cover plate has an 80 - $\mu \mathrm{m}$-diameter pinhole and windows for passing irradiated laser beams and is made of a $50-\mu \mathrm{m}$-thick SUS plate coated with black oxide film. Irradiated laser beams are scattered, Doppler shifted by blood cells, pass through the pinhole, and then detected by the PD in the $\mu$-LDV. The pinhole restricts the entry of higher multiply scattered light into the PD active area, because multiple scattered light broadens the power spectrum and obscures the beat signal peak. broaden the power spectrum and obscure the beat signal peak. The detected scattered light coming from the center of the tube becomes higher in intensity than the higher multiply scattered light after setting the cover plate.

A PTFE tube was used as the flow channel with inner and outer diameters of 0.5 and 1.0 $\mathrm{mm}$, respectively. The $\mu$-LDV was set in front of the flow tube, as shown in Fig. 3. The gap between the $\mu$-LDV surface and the tube surface was set to $0.3 \mathrm{~mm}$ so that the intersection of 


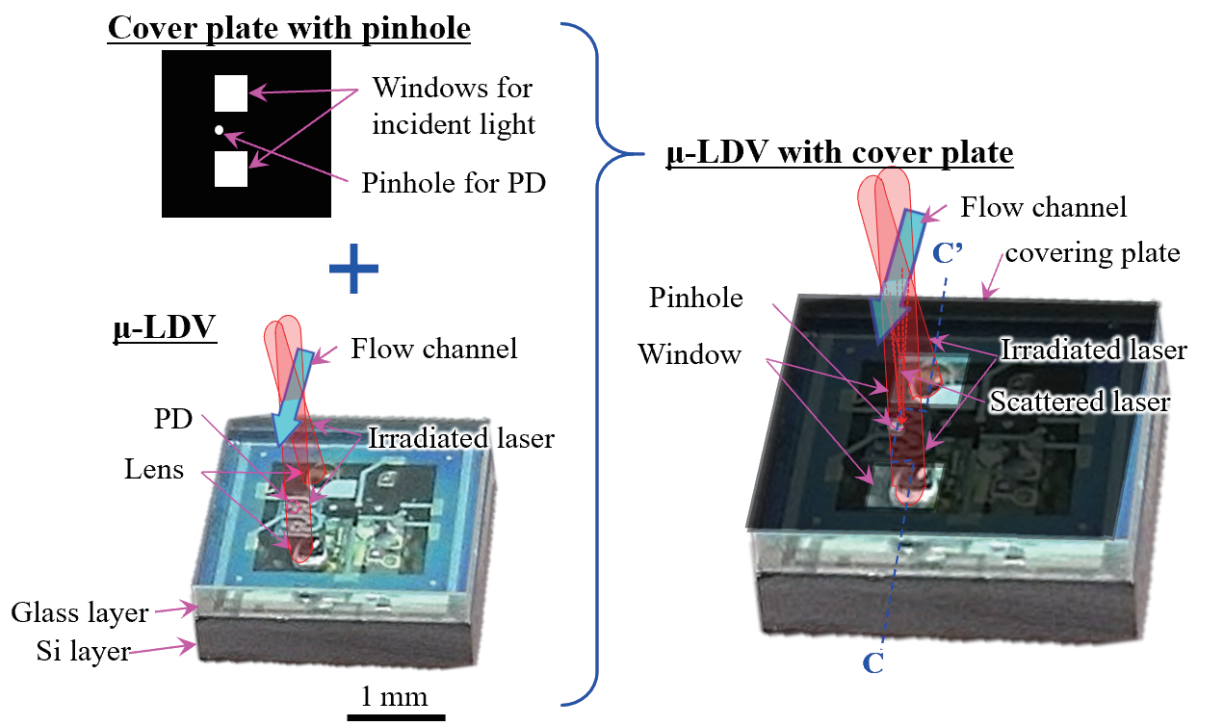

Fig. 2. (Color online) $\mu$-LDV and cover plate with a pinhole for PD.

Table 1

Specifications of the $\mu$-LDV.

\begin{tabular}{ccccccc}
\hline $\begin{array}{c}\text { Laser } \\
\text { wavelength }\end{array}$ & $\begin{array}{c}\text { Laser input } \\
\text { current }\end{array}$ & $\begin{array}{c}\text { Laser beam } \\
\text { power }\end{array}$ & $\begin{array}{c}\text { Laser beam } \\
\text { diameter }\end{array}$ & $\begin{array}{c}\text { Half-angle of two } \\
\text { crossing laser beams }\end{array}$ & $\begin{array}{c}\text { PD active } \\
\text { diameter }\end{array}$ & PD responsivity \\
\hline $1310 \mathrm{~nm}$ & $30 \mathrm{~mA}$ & $\begin{array}{c}2.5 \mathrm{~mW} \\
\text { (one side) }\end{array}$ & $\begin{array}{c}0.3 \mathrm{~mm} \\
\text { (FWHM) }\end{array}$ & 23.8 degree & $70 \mu \mathrm{m}$ & $\begin{array}{c}0.9 \mathrm{~A} / \mathrm{W} \\
\text { (at } 1310 \mathrm{~nm})\end{array}$ \\
\hline
\end{tabular}
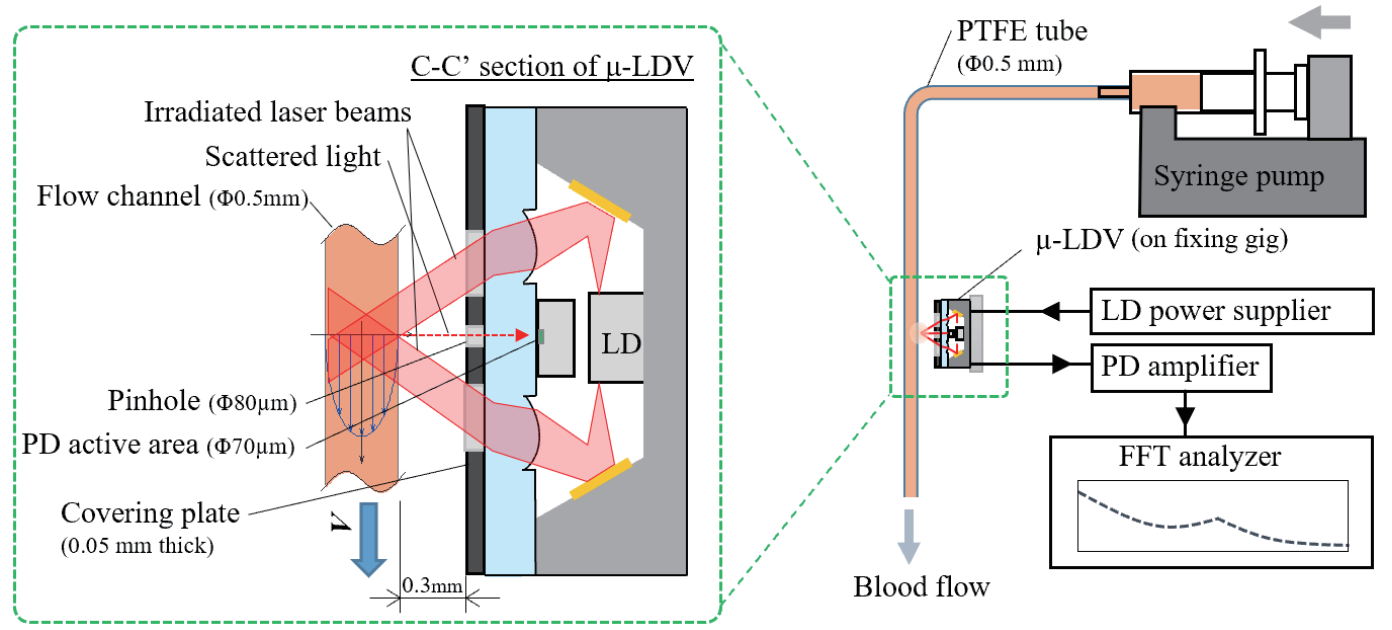

Fig. 3. (Color online) Experimental setup and schematic of C-C' section of $\mu$-LDV with flow channel.

the center of the two laser beams is located at the center of the tube. Blood (defibrinated horse blood, Cohjin Bio Co., Ltd.) was transferred with a syringe pump at flow rates of 58.9, 235.6, and $471.1 \mu \mathrm{l} / \mathrm{min}$, which correspond to the theoretical flow velocities at the center of the flow channel, $V_{t c}$, of 10,40 , and $80 \mathrm{~mm} / \mathrm{s}$, respectively, under the assumption of Newtonian flow, 
which follows the Hagen-Poiseuille law. The PD output signal of the $\mu$-LDV was amplified 5 times, and the processing parameters of the fast Fourier transform (FFT) are listed in Table 2.

Hematocrit was adjusted to 20,40 , and $60 \%$ by mixing autologous plasma and RBCs that were prepared beforehand by centrifugation of the blood. These blood samples are shown in Fig. 4. Blood samples with lower hematocrit values appears brighter red. The optical transmission was low enough that the background could not be observed through blood samples.

\subsection{Conversion theory from beat frequency to flow rate}

Figure 5 illustrates the conversion theory from beat frequency to flow rate. Fluid flows in a tube of section area $A$ at flow rate $Q_{a c t}$ and average velocity $V_{a v e}$ in accordance with

$$
Q_{a c t}=A V_{\text {ave }}
$$

Here, assuming that the flow velocity distribution for the radial direction $r, V_{(r)}$, follows the Hagen-Poiseuille law, the relationship between velocity at the center of the tube $V_{(r=0)}$ and $V_{\text {ave }}$ is expressed as

Table 2

FFT parameters.

\begin{tabular}{ccccccc}
\hline $\begin{array}{c}\text { FFT } \\
\text { range }\end{array}$ & $\begin{array}{c}\text { Sampling } \\
\text { rate }\end{array}$ & $\begin{array}{c}\text { No. of samplings } \\
\text { for one FFT period }\end{array}$ & $\begin{array}{c}\text { No. of frequency } \\
\text { divisions }\end{array}$ & $\begin{array}{c}\text { Resolution } \\
\text { bandwidth }\end{array}$ & Averaging & $\begin{array}{c}\text { Measurement } \\
\text { time }\end{array}$ \\
\hline $100 \mathrm{kHz}$ & $256 \mathrm{kHz}$ & 2048 points & 800 & $125 \mathrm{~Hz}$ & $\begin{array}{c}100 \text { times, } \\
90 \% \text { overlap }\end{array}$ & $87.2 \mathrm{~ms}$ \\
\hline
\end{tabular}

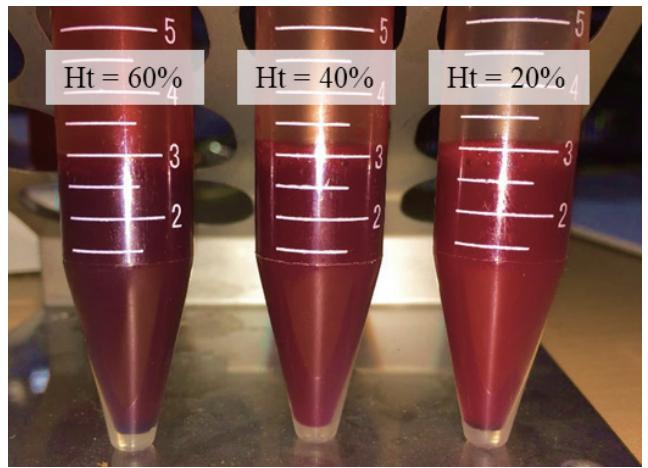

Fig. 4. (Color online) Blood samples with different hematocrit.

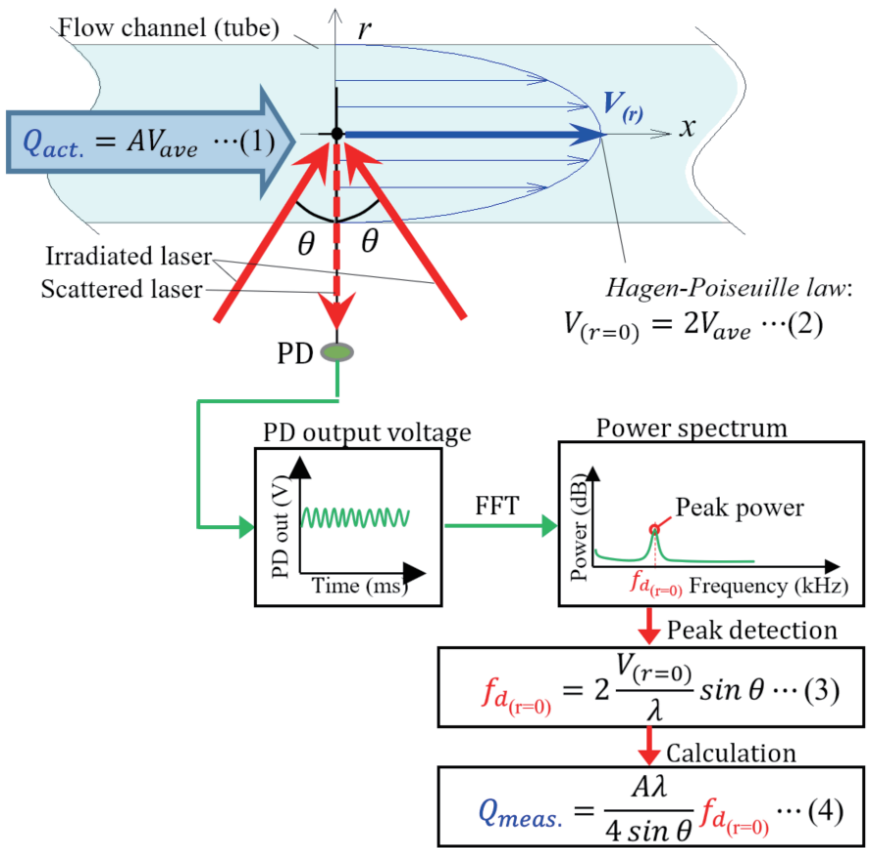

Fig. 5. (Color online) Conversion process for measurement of absolute flow rate in a tube by laser Doppler velocimetry. 


$$
V_{(r=0)}=2 V_{\text {ave }}
$$

The LDV irradiates two laser beams at the center of fluid flow, then the PD detects light scattered and Doppler shifted by particles (i.e., RBCs). The output voltage is analyzed by FFT. Then, the power spectrum gives the beat frequency $f_{d(r=0)}$ as the frequency at the peak power of the power spectrum. $f_{d(r=0)}$ is expressed as

$$
f_{d(r=0)}=2 V_{(r=0)} \sin \theta / \lambda,
$$

where $\lambda$ is the wavelength of the laser and $\theta$ is the half-angle between two crossing laser beams. From these relations, the flow rate $Q_{\text {meas. }}$ can be calculated from the measured beat frequency as

$$
Q_{\text {meas }}=A \lambda f_{d(r=0)} /(4 \sin \theta) \text {. }
$$

\subsection{Signal processing}

Ideally, the velocity at the center of a tube can be perfectly measured if it is possible to irradiate extrafine collimated laser beams as schematically shown in Fig. 5. In practice, however, it is a challenge to irradiate the laser at such a specific volume owing to high scattering ability of the large number of RBCs in blood. Moreover, forming an extrafine collimated laser beam for the 0.5 -mm-diameter tube (i.e., 20 - $\mu \mathrm{m}$-diameter beam) is also challenging without a long optical path, even in a uniform medium with low scattering ability such as air. Thus, the obtained power spectra include a wide band of Doppler-shifted frequency corresponding to velocity distribution in the measurement volume of the LDV. Therefore, under the measurement conditions adopted in this study, the power spectrum obtained by laser Doppler velocimetry never shows sharp peaks, as schematically shown in Fig. 5. Preprocessing is necessary to enable the detection of the beat signals corresponding to the center velocity, which are buried in the broad power spectrum. We propose frequency weighting modification of the power spectrum, as defined in Eqs. (5) and (6), to reveal the buried beat frequency.

$$
\begin{gathered}
P_{i(f i)}=20 \log _{10}\left(V_{i(f i)}\right) \\
P_{i(f i)}^{\prime}=20 \log _{10}\left\{V_{i(f i)}\left\{\left(f_{i}+\beta f_{\text {max }}\right) /\left[(1+\beta) f_{\text {max }}\right]\right\}^{\alpha}\right\}
\end{gathered}
$$

Here, $f_{i}, V_{i(f i)}, P_{i(f i)}, P_{i(f i)}^{\prime}, f_{\max }, \alpha$, and $\beta$ are the frequency, power at $f_{i}$ in volts, power at $f_{i}$ in $\mathrm{dB}$, modified power at $f_{i}$ in $\mathrm{dB}$, maximum frequency of FFT, frequency weighting coefficient, and offset frequency ratio, respectively. Subscript $i$ indicates the element number of the FFTanalyzed data array. Equation (6) can be rearranged as

$$
P_{i(f i)}^{\prime}=P_{i(f i)}+20 \alpha \log _{10}\left(f_{i}+\beta f_{\max }\right)-20 \alpha \log _{10}\left[(1+\beta) f_{\max }\right]
$$


The sequence of beat frequency detection is described in Fig. 6. First, the raw power spectrum is modified as described by Eq. (7). Then, the modified power spectrum shows an arched peak point. After that, peak detection is performed by comparing modified powers, following the sequence described in Fig. 6. $f_{i}$ is scanned from $0 \mathrm{~Hz}$ until the peak is found. $f_{i}$ is detected as a beat frequency $f_{\text {d.meas }}$ if the power at $f_{i}$ is higher than all the powers at frequencies in the range of $f_{i}$ to $f_{i}+10 \mathrm{kHz}\left(=f_{i}+f_{i+80}\right)$. Flow rate can be calculated using $f_{\text {d.meas }}$ and Eq. (4). We named the above flow rate measurement process the frequency weighting method. The error for the theoretical beat frequency $E_{F W}$ is defined as follows using the theoretical beat frequency at the center $f_{d(r=0)}$ :

$$
E_{F W}=\left(f_{\text {d.meas }(r=0)}-f_{d(r=0)}\right) / f_{d(r=0)} .
$$

Additionally, an arbitrary value related to the blood flow rate, blood flow value $(B F V)$ measured by the LDF method [Eq. (9)], ${ }^{(35)}$ was used for comparing measurement errors in the frequency weighting method and the LDF method.

$$
B F V=\left(\sum_{i=1}^{n} P_{i(f i)} f_{i} d f\right) /\left(\sum_{i=1}^{n} P_{i(f i)} d f\right)
$$

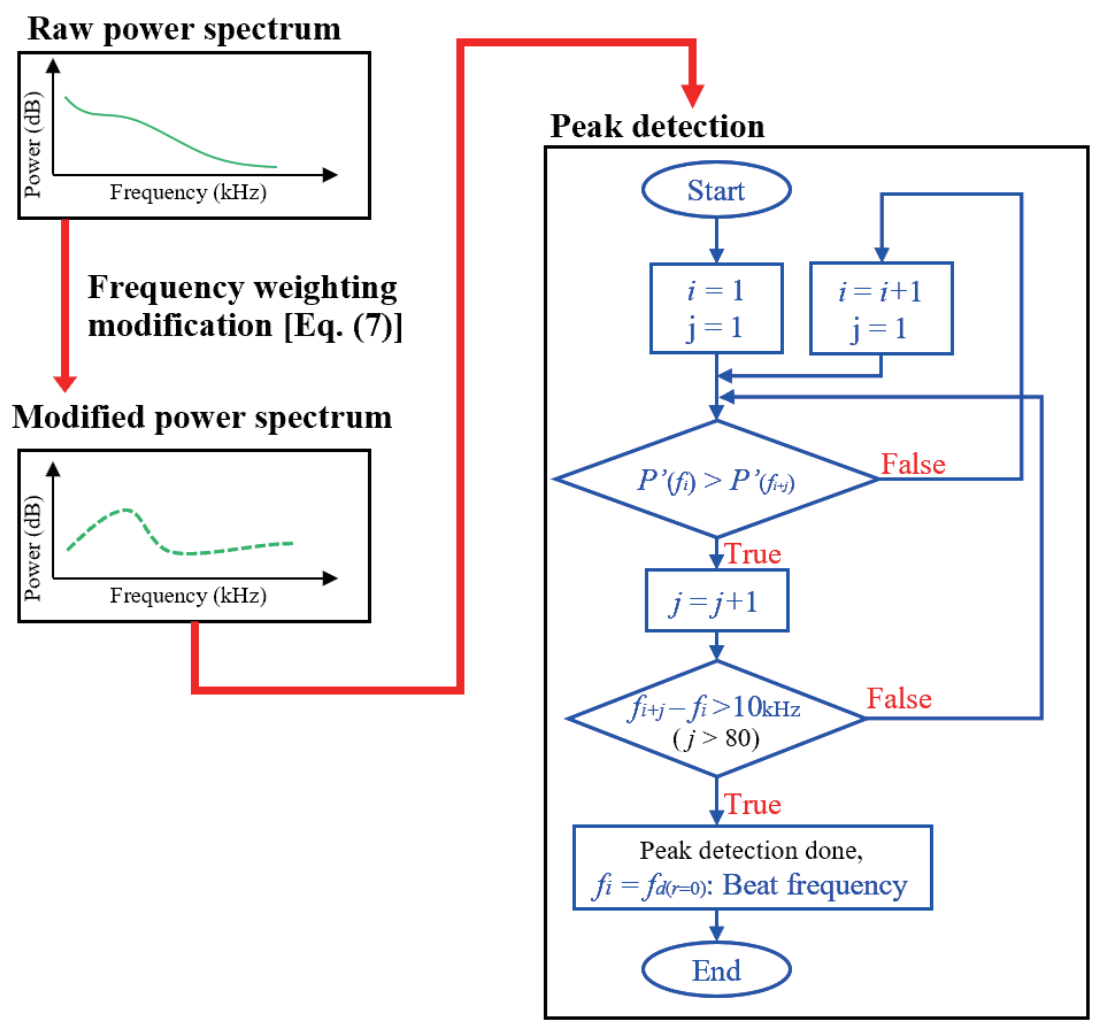

Fig. 6. (Color online) Sequence of beat frequency detection. 
The measurement error in the LDF method $E_{L D F}$ is defined as follows. From average $B F V$ at $V_{c t}$ for each hematocrit, the approximate straight line of average $B F V, B F V_{\text {approx. }}$, is calculated by the least squares method.

$$
B F V_{\text {approx. }(V c t)}=a V_{c t}+b
$$

$a$ and $b$ are constant coefficients. $E_{L D F}$ is defined as

$$
E_{L D F}=\left(B F V-B F V_{\text {approx. }(V c t)}\right) /\left[\left(B F V_{\text {approx. }(V c t)}\right)-b\right]
$$

\section{Results and Discussion}

Figures 7 (a) -7 (c) show power spectra of the $\mu$-LDV while changing 20,40 , and $60 \%$ hematocrit at flow rates of $58.9 \mu 1 / \mathrm{min}\left(V_{t c}=10 \mathrm{~mm} / \mathrm{s}\right), 235.6 \mu 1 / \mathrm{min}\left(V_{t c}=40 \mathrm{~mm} / \mathrm{s}\right)$, and $471.1 \mu \mathrm{l} / \mathrm{min}\left(V_{t c}=80 \mathrm{~mm} / \mathrm{s}\right)$. The shape of the power spectrum became wider toward higher frequencies as the flow rate increased. A wide spectral shape was observed in the higher frequency region as flow rate increased for all investigated hematocrit values. However, these power spectra have no specific peak, as illustrated in Fig. 5. Therefore, we applied the frequency weighting method as proposed in the previous section. Using the measured data, the coefficients $\alpha$ and $\beta$ in Eq. (7) were optimized so that the change in the measured value was the smallest when the hematocrit was varied. The optimized coefficients $\alpha$ and $\beta$ were 0.9 and 0.01 , respectively. The modified power spectra are shown in Figs. 7(d)-7(f). All modified power spectra have rounded peak points. The detected peak points are shown in the graph as inverse triangles $(\boldsymbol{\nabla})$. Also, the detected beat frequencies are plotted with the theoretical beat frequency for theoretical velocity at the center [Eq. (3)] in Fig. 8. The detected beat frequency linearly changes along the theoretical beat frequency. In particular, the detected beat frequencies at the same velocity have small errors with respect to the wide range of hematocrit change from 20 to $60 \%$, which is the range that fully covers the range of human blood hematocrit values (i.e., 40 to $54 \%$ for men, 36 to $48 \%$ for women ${ }^{(32)}$ ). These errors in the frequency weighting method are summarized in Table 3. The errors with respect to hematocrit changes for each flow velocity at the center were $14.2 \%$ for $10 \mathrm{~mm} / \mathrm{s}(58.9 \mu \mathrm{l} / \mathrm{min}), 5.1 \%$ for $40 \mathrm{~mm} / \mathrm{s}(235.6 \mu 1 / \mathrm{min})$, and $3.6 \%$ for $80 \mathrm{~mm} / \mathrm{s}(471.1 \mu \mathrm{l} / \mathrm{min})$. We considered the error to be caused by the deformation of the power spectrum shape owing to variation in the concentration of RBCs, which are the main scatterer and absorber in the blood. Since higher-hematocrit blood has higher scattering ability and absorbability, the frequency distribution of the power spectrum becomes broader and the total intensity of the power spectrum becomes lower. In addition, the penetration depth of light is smaller, resulting in the intensity of the received light coming from the vicinity of the LDV being higher than the intensity from the central portion of the flow channel. As a result, the intensity of the Doppler-shifted light due to the blood having a lower flow rate than the blood in the central portion of the channel increases, and not only the decrease in the power spectrum intensity but also the frequency distribution are affected. 


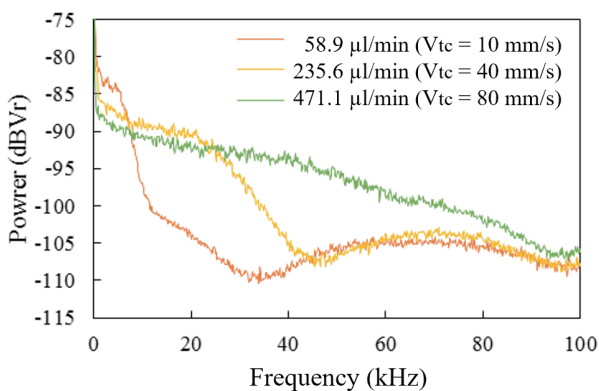

(a)

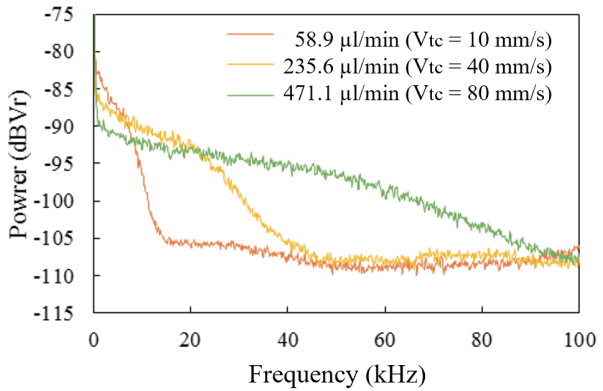

(c)

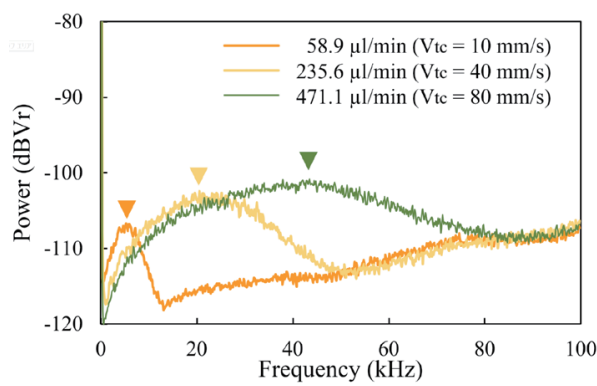

(e)

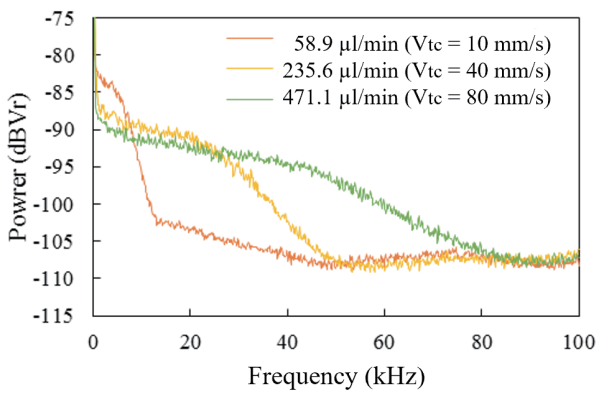

(b)

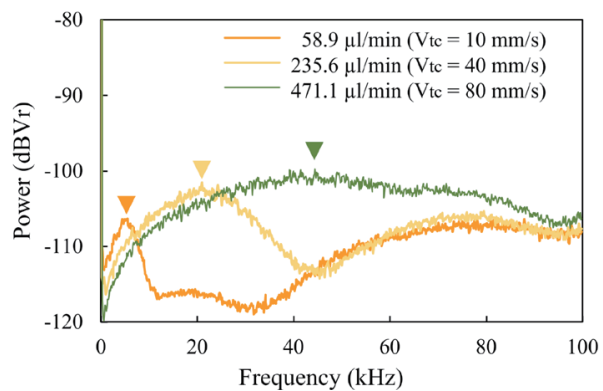

(d)

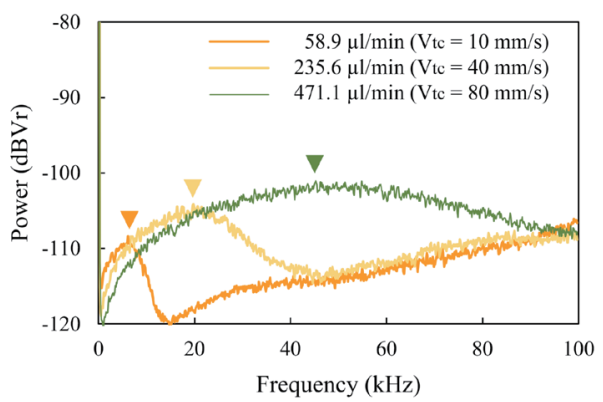

(f)

Fig. 7. (Color online) Power spectra of $\mu$-LDV output signal during flow measurement. (a)-(c) Raw power spectra. (d)-(f) Modified power spectra. Detected peaks are marked by $\boldsymbol{\nabla}$. (a) $\mathrm{Ht}=60 \%$. (b) $\mathrm{Ht}=40 \%$. (c) $\mathrm{Ht}=20 \%$. (d) $\mathrm{Ht}=60 \%$. (e) $\mathrm{Ht}=40 \%$. (f) $\mathrm{Ht}=20 \%$.

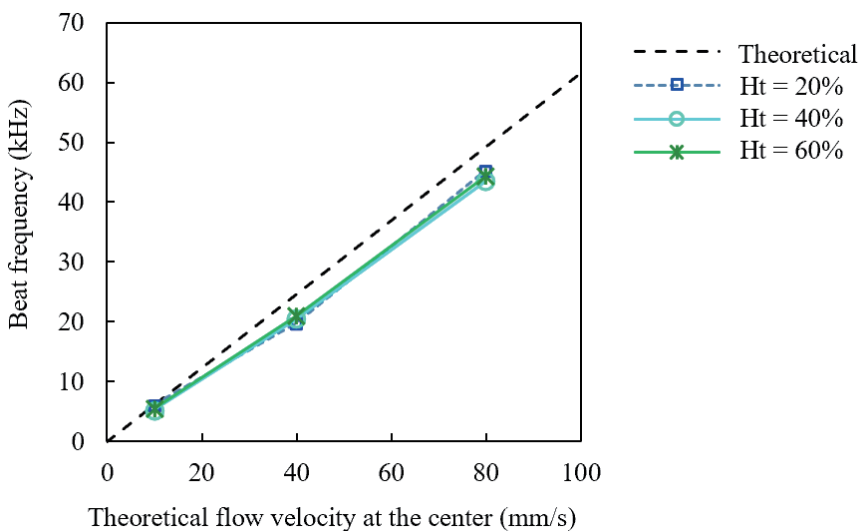

Fig. 8. (Color online) Detected beat frequencies and theoretical beat frequency for theoretical velocity at the center. 
Table 3

Measurement errors in frequency weighting method.

\begin{tabular}{lrrrr}
\hline Flow velocity & \multicolumn{2}{l}{ Error for the theoretical beat frequency (\%) } & \multicolumn{2}{c}{$\begin{array}{c}\text { Error with respect } \\
\text { to hematocrit change (\%) }\end{array}$} \\
\cline { 2 - 4 } at the center $(\mathrm{mm} / \mathrm{s})$ & $\mathrm{Ht} 20$ & $\mathrm{Ht} 40$ & $\mathrm{Ht} 60$ & 14.2 \\
\hline 10 & -0.5 & -14.7 & -10.6 & 5.1 \\
40 & -19.8 & -16.7 & -14.7 & 3.6 \\
80 & -8.1 & -11.6 & -9.9 & \\
\hline
\end{tabular}

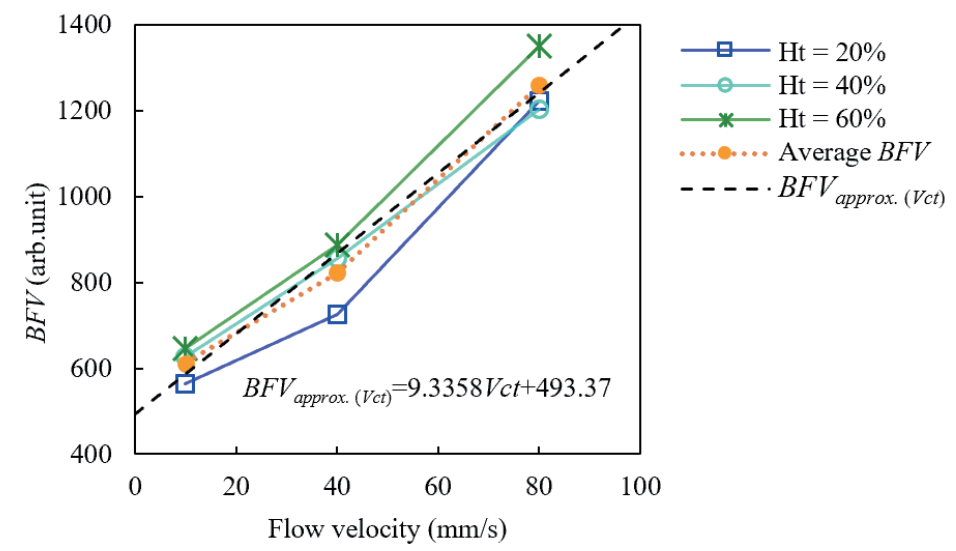

Fig. 9. (Color online) Arbitrary BFV determined by LDF calculation [Eq. (9)].

Table 4

Measurement errors in LDF method.

\begin{tabular}{|c|c|c|c|c|}
\hline \multirow{2}{*}{$\begin{array}{l}\text { Flow velocity } \\
\text { at the center }(\mathrm{mm} / \mathrm{s})\end{array}$} & \multicolumn{3}{|c|}{ Error for theoretical beat frequency (\%) } & \multirow{2}{*}{$\begin{array}{l}\text { Error with respect } \\
\text { to different hematocrit levels }\end{array}$} \\
\hline & Ht 20 & Ht 40 & Ht 60 & \\
\hline 10 & -25.1 & 41.4 & 64.1 & 89.2 \\
\hline 40 & -37.5 & -2.8 & 5.2 & 42.7 \\
\hline 80 & -2.3 & -4.8 & 14.7 & 19.5 \\
\hline
\end{tabular}

$B F V$ and its errors were also calculated using Eqs. (9)-(11) to compare the measurement errors. Calculated $B F V$, average $B F V$ at each flow rate, and $B F V_{\text {approx.(Vct) }}$ are plotted in Fig. 9. Obviously, $B F V$ indicated different values when hematocrit was different under the same flow rate condition. These errors in the LDF method are summarized in Table 4. The errors with respect to the hematocrit change for each flow velocity at the center were $89.2 \%$ for $10 \mathrm{~mm} / \mathrm{s}(58.9 \mu 1 / \mathrm{min}), 42.7 \%$ for $40 \mathrm{~mm} / \mathrm{s}(235.6 \mu \mathrm{l} / \mathrm{min})$, and $19.5 \%$ for $80 \mathrm{~mm} / \mathrm{s}(471.1 \mu 1 / \mathrm{min})$, all of which are larger than the errors in the frequency weighting method. These results show the advantage of $\mu$-LDV with the frequency weighting method for flow rate measurement of unknown hematocrit. The error in the LDF method is thought to be due to the issue that highhematocrit blood decreases the linearity of the LDF method. ${ }^{(36)}$ As Fig. 9 indicates, the error seems to be more dependent on hematocrit than flow velocity; the relative error becomes larger as the speed decreases. 
For further improvement in measurement accuracy, there is still the following need. Because the power spectrum has noise, the current method based principally on peak detection could have measurement error. Considering the noise level of the result, the error can be approximately 5 times of the frequency resolution at maximum $(625 \mathrm{~Hz})$, which corresponds to $1 \mathrm{~mm} / \mathrm{s}$, and $10 \%$ error of $V_{c t}$ at $10 \mathrm{~mm} / \mathrm{s}$. This error can be reduced by applying appropriate curve fitting to the power spectrum for the interpolation of the discrete frequency data. Another possible solution is to improve the frequency resolution or increase the number of averaging times, $N$. A higher frequency resolution leads to a higher measurement resolution, and increasing the number of averaging times can reduce the noise to be inversely proportional to root $N$ under the assumption that the noise is random. The disadvantage of these two methods is a decrease in response speed. The frequency division of FFT is inversely proportional to the sampling rate, or increasing $N$ simply results in the sampling times becoming $N$ times. However, since the flow in microfluidic devices does not rapidly change owing to its low Reynold's number, it is considered that the technique of improving the accuracy at the expense of the response speed may be effective in practice.

Additionally, we assumed the flow velocity distribution to be Newtonian. However, blood is a non-Newtonian fluid and its flow shows complex biorheological behavior, including RBC aggregation. ${ }^{(37)}$ The flow velocity distribution and RBC behavior should be considered for better accuracy.

\section{Conclusions}

We investigated the validity and application of the $\mu$-LDV integrated into a microfluid device developed by our group for absolute blood flow measurement. It offers the important function of precise flow control. We proposed a frequency weighting method with a signal processing algorithm that enables beat frequency detection from a power spectrum that is broad and weak owing to marked light scattering and absorption by RBCs. The results of measurement by the proposed method showed that the measurement error due to hematocrit change could be significantly reduced compared with the LDF method. In addition, the LDV can measure the flow velocity and flow rate of various lightscattering fluids accurately without contact and in real time. Owing to the millimeter-scale size, the addition of the proposed signal processing, and the advantages of LDV, the $\mu$-LDV has the potential to be an integrable absolute flow rate sensor for LoCs and $\mu$-TASs for various fluids.

\section{Acknowledgments}

Part of this work was supported by JSPS KAKENHI, Grant Number JP16J04014.

\section{References}

1 E. K. Sackmann, A. L. Fulton, and D. J. Beebe: Nature 507 (2014) 181.

2 H. Becker and L. E. Locascio: Talanta 56 (2002) 267. 
3 J. W. Zhou, A. V. Ellis, and N. H. Voelcker: Electrophoresis 31 (2010) 2.

4 X. Y. Ding, P. Li, S. C. S. Lin, Z. S. Stratton, N. Nama, F. Guo, D. Slotcavage, X. L. Mao, J. J. Shi, F. Costanzo, and T. J. Huang: Lab Chip 13 (2013) 3626.

5 A. A. Yazdi, A. Popma, W. Wong, T. Nguyen, Y. Pan, and J. Xu: Microfluid. Nanofluid. 20 (2016) 1.

6 R. D. Sochol, E. Sweet, C. C. Glick, S. Y. Wu, C. Yang, M. Restaino, and L. W. Lin: Microelectron. Eng. 189 (2018) 52.

7 A. Kecskemeti and A. Gaspar: Anal. Chim. Acta 1021 (2018) 1.

8 I. Rodriguez-Ruiz, V. Babenko, S. Martinez-Rodriguez, and J. A. Gavira: Analyst 143 (2018) 606.

9 R. R. Sathuluri, Y. S. Kurniawan, J. Y. Kim, M. Maeki, W. Iwasaki, S. Morisada, H. Kawakita, M. Miyazaki, and K. Ohto: Sep. Sci. Technol. 53 (2018) 1261.

10 I. J. Jahn, O. Zukovskaja, X. S. Zheng, K. Weber, T. W. Bocklitz, D. Cialla-May, and J. Popp: Analyst 142 (2017) 1022.

11 V. I. Furdui and D. J. Harrison: Lab Chip 4 (2004) 614.

12 M. Mir, A. Homs, and J. Samitier: Electrophoresis 30 (2009) 3386.

13 J. Yoon, M. K. Park, T. Y. Lee, Y. J. Yoon, and Y. Shin: Lab Chip 15 (2015) 3530.

14 S. Grünzner, F. V. Reddavide, C. Steinfelder, M. Cui, M. Busek, U. Klotzbach, Y. Zhang, and F. Sonntag: SPIE BiOS 10061 (2017) 13.

15 N. Khalid, I. Kobayashi, and M. Nakajima: WIREs-Syst. Biol. Med. 9 (2017) e1381.

16 Y. S. Sun: Sensors 17 (2017) 2048.

17 J. D. Wu, M. L. Dong, S. Santos, C. Rigatto, Y. Liu, and F. Lin: Sensors 17 (2017) 2934.

18 J. Ozhikandathil, S. Badilescu, and M. Packirisamy: J. Neural Transm. 124 (2017) 47.

19 V. Lien, K. Zhao, Y. Berdichevsky, and Y. H. Lo: IEEE J. Selected Topics in Quantum Electronics 11 (2005) 827.

20 J. Godin, V. Lien, and Y.-H. Lo: Appl. Phys. Lett. 89 (2006) 061106.

21 H. Ernst, A. Jachimowicz, and G. A. Urban: Sens. Actuators, A 100 (2002) 54.

22 J. Collins and A. P. Lee: Lab Chip 4 (2004) 7.

23 G. B. Lee, T. Y. Kuo, and W. Y. Wu: Exp. Therm. Fluid Sci. 26 (2002) 435.

24 E. J. Carboni, B. H. Bognet, G. M. Bouchillon, A. L. Kadilak, L. M. Shor, M. D. Ward, and A. W. K. Ma: Biophys. J. 111 (2016) 1487.

25 L. Stern, A. Bakal, M. Tzur, M. Veinguer, N. Mazurski, N. Cohen, and U. Levy: Sensors 14 (2014) 16799.

26 T. Tanaka, C. Riva, and I. Ben-Sira: Science 186 (1974) 830.

27 P. Aarts, S. A. T. Vandenbroek, G. W. Prins, G. D. C. Kuiken, J. J. Sixma, and R. M. Heethaar: Arteriosclerosis 8 (1988) 819.

28 L. Campagnolo, S. Roman, J. Perchoux, and S. Lorthois: Comput. Methods Biomech. Biomed. Eng. 15 (2012) 104.

29 K. T. Kotz, W. Xiao, C. Miller-Graziano, W.-J. Qian, A. Russom, E. A. Warner, L. L. Moldawer, A. De, P. E. Bankey, B. O. Petritis, D. G. Camp, A. E. Rosenbach, J. Goverman, S. P. Fagan, B. H. Brownstein, D. Irimia, W. Xu, J. Wilhelmy, M. N. Mindrinos, R. D. Smith, R. W. Davis, R. G. Tompkins, M. Toner, the Inflammation and the Host Response to Injury Collaborative Research Program: Nat. Med. 16 (2010) 1042.

30 K. Loutherback, J. D'Silva, L. Liu, A. Wu, R. H. Austin, and J. C. Sturm: AIP Adv. 2 (2012) 042107.

31 T. Cochrane, J. Earnshaw, and A. Love: Med. Biol. Eng. Comput. 19 (1981) 589.

32 H. H. Billett: Clinical Methods: The History, Physical, and Laboratory Examinations (1990) Chap. 151.

33 N. Morita, H. Nogami, E. Higurashi, and R. Sawada: Sensors 18 (2018) 326.

34 N. Morita, H. Nogami, E. Higurashi, T. Ito, and R. Sawada: J. Microelectromech. Syst. 25 (2016) 380.

35 R. N. R. Bonner: Appl. Opt. 20 (1981).

36 V. Rajan, B. Varghese, T. van Leeuwen, and W. Steenbergen: Lasers Med. Sci. 24 (2009) 269.

37 S. Roman, S. Lorthois, P. O. Duru, and F. Risso: Microvasc. Res. 84 (2012) 249. 